Derek M. Tole

Cathryn L. Edrich

Bruce A. Noble

Department of Ophthalmology

Clarendon Wing

The General Infirmary at Leeds

Belmont Grove

Leeds LS2 9NS, UK

Miss C.L. Edrich

66 Woodcross Fold

Morley

Leeds LS27 9JR, UK

Sir,

\section{Actinic keratoconjunctivitis and minocycline}

Minocycline is widely used as second-line therapy for acne vulgaris. It is given as a once- or twice-daily preparation and the antibiotic appears not to induce resistance. Although this tetracycline is found to be efficacious in the management of acne, it is associated with a recognised profile of side-effects. Infrequently, the eye is adversely affected by minocycline.

We present the case of a patient who developed acute actinic keratoconjunctivitis secondary to minocycline use. To our knowledge this is the first report of such an interaction.

\section{Case report}

A 21-year-old Caucasian man with a 1 day history of bilateral eye discomfort associated with watering, photophobia and blurred vision was seen by his general practitioner and treated with co-amoxiclav, ibuprofen and topical fusidic acid. The patient had been in bright sunshine for a short period the previous day. He had not performed any recent arc-welding, snow-skiing or boating. He had started oral minocycline 2 weeks earlier for acne vulgaris.

The patient re-presented a day later as his symptoms of eye pain, watering and blurred vision became progressively worse. He had intense photophobia and marked blepharospasm. The visual acuities were $6 / 18$ right and 6/4 left unaided, the right improving to $6 / 6$ with pinhole. Diffuse punctate epitheliopathy was seen affecting the entire cornea in both eyes, along with marked papillary conjunctivitis, reminiscent of the findings in a person who has been arc-welding for a prolonged period of time without protective eyewear. A florid photosensitivity skin eruption was seen on the face, anterior trunk and flexor surfaces of the upper arms. A degree of facial angioedema was noted. He was asked to stop minocycline and the epitheliopathy was treated with chloramphenicol ointment.

Vision returned to normal within 2 days, and the eyes became asymptomatic within 4 days with complete resolution of the punctate epitheliopathy. The skin reaction resolved in 1 week.
Comment

The onset of the diffuse punctate epithelial keratitis along with the cutaneous photosensitivity reaction at lightexposed areas of the face and trunk 2 weeks after starting systemic minocycline and its subsequent improvement on cessation of the drug is highly suggestive of a causeand-effect relationship. It is known that the resolution of photosensitivity may occur with either protection from the relevant radiation or drug withdrawal. ${ }^{1}$ Rechallenge with the drug was felt to be unethical considering the distressing nature of the symptoms.

The mechanism of photosensitivity remains unclear; ${ }^{2}$ however, it has been noted that some tetracyclines induce greater degrees of photosensitivity than others. Minocycline has been regarded as the least phototoxic agent, ${ }^{1,3}$ whereas doxycycline is a more potent photosensitiser. ${ }^{4}$ Some authors have even indicated that minocycline has virtually no phototoxic effect. ${ }^{5,6}$

Photosensitivity implies a reaction to usually harmless ultraviolet or visible radiation doses. Drugs may induce photosensitivity either by direct mechanisms where the drug is present in the affected organ unaltered or altered, or through indirect effects as is seen in drug-associated lupus erythematosus or hepatic porphyria. Direct reactions comprise the two broad categories of phototoxic and photoallergic outcomes.

Phototoxic reaction is seen with the anti-arrythmic drug amiodarone, whose chemical structure is closely related to the psoralens. Other drugs with similar reactions include the non-steroidal anti-inflammatory compounds benoxaprofen and azapropazone, the tricyclic antidepressant protriptyline, the retinoid etretinate, the thiazide diuretics hydrochlorothiazide and cyclopenthiazide, fluorouracil, vinblastine, and the psoralen 8-methoxypsoralen.

Photoallergic reactions are mediated via the immune response. Chlorpromazine, quinine and quinidine are systemic drugs that have demonstrated this reaction. Halogenated phenols such as tetrachlorosalicylanilide and buclosamide can exert topical photoallergic responses, as can para-aminobenzoic acid esters, which are found in sunscreen lotions.

The action spectrum of most drug photosensitisers, including tetracyclines, is within the solar UVA range $(320-400 \mathrm{~nm})$. These frequencies of radiation, unlike UVB, are not filtered by window glass.

Minocycline is routinely prescribed for acne vulgaris and its side-effect profile is well known. It can cause a range of effects including gastrointestinal disturbances, autoimmune hepatitis and systemic lupus erythematosus (SLE)-like syndrome, ${ }^{7}$ vestibular disturbances and pigmentation of many organs, including the $\operatorname{skin}^{8}$ and sclerae. ${ }^{9}$

Since 1 July 1963 the Medicines Control Agency in the United Kingdom has received notification of nearly 2000 instances of adverse reactions with minocycline. ${ }^{10}$ Minocycline has been associated with a number of ocular conditions. These include single cases of dry eyes, conjunctivitis, eyelid swelling and cataract. Eight patients 
developed abnormal vision, 3 suffered eye discomfort and 1 had tired eyes. One 57-year-old patient experienced a photosensitive facial rash with conjunctival reaction after sitting in the sun following administration of a single tablet of minocycline. There have been a total of 512 recorded cutaneous reactions to date associated with minocycline, including 100 cases of rash, 25 cases of photosensitivity eruption, 45 cases of angioedema and 41 cases of bullous dermatoses. One patient was reported to have suffered an intense photosensitive facial reaction around the eyes and trunk to a degree that the irritation prevented her from leaving her house for the duration of the symptoms.

Whilst ocular effects of minocycline are rare, we would recommend that patients being started on such treatment be advised to seek ophthalmic advice if ocular symptoms occur. Although this represents the first documented case of minocycline-induced actinic keratoconjunctivitis it may be reported more frequently in the future with the increased use of minocycline.

\section{References}

1. Smith.AG. Drug-induced photosensitivity. Adv Drug Reaction Bull 1989;136:508-11.

2. Hasan T. Mechanism of tetracycline photosensitivity. J Invest Dermatol 1984;83:179-83.

3. Dukes MNG, editor. Mylers side effects of drugs. 9th ed. Amsterdam: Excerpta Medica, 1980:342-433.

4. Frost $P$, et al. Phototoxic potential of minocycline and doxycycline. Arch Dermatol 1972;105:681-3.

5. Ljunggrun B. Systemic drug photosensitivity. Photodermatology 1986;3:26-36.

6. Hasan T, et al. Mechanism of tetracycline phototoxicity. J Invest Dermatol 1984;83:179-83.

7. Gough A, Chapman S, Wagstaff K, Emery P, Elias E. Minocycline induced autoimmune hepatitis and systemic lupus erythematosus-like syndrome. BMJ 1996;312:169-72.

8. Goulden V, Glass D, Cunliffe WJ. Safety of long-term highdose minocycline in the treatment of acne. BJ Dermatol 1996;134:693-5.

9. Sabroe RA, Archer CB, Harlow D, Bradfield JW, Peachey RD. Minocycline-induced discoloration of the sclerae. $\mathrm{Br} \mathrm{J}$ Dermatol 1996;135:314-6.

10. Adverse Drug Reactions On-line Information Tracing (ADROIT) drug analysis print. Medicine Control Agency, Market Towers, 1 Nine Elms Lane, London SW8 5NQ.

Waqaar Shah

R. De Cock

Surgical Services Directorate

Ophthalmology Department

Kent and Canterbury Hospital

Ethelbert Road

Canterbury

Kent CT1 3NG, UK

Sir,

\section{Chiasmal apoplexy, an unusual complication of cerebral glioblastoma}

The differential diagnosis of the rapid development of bilateral visual failure includes optic glioma. ${ }^{1,2}$ and meningeal carcinomatosis. ${ }^{3}$ We now describe a case of cerebral glioblastoma complicated by infarction of the optic chiasm.

\section{Case report}

A 70-year-old woman with no past medical history of note initially presented to the physicians with symptoms and signs consistent with a left hemispheric cerebrovascular accident. A right carotid bruit was present, her blood pressure was elevated (200/90 $\mathrm{mmHg}$ ) and she was commenced on metoprolol and aspirin. At this time her visual functions were intact.

Ten weeks later, over a $12 \mathrm{~h}$ period she developed profound bilateral visual loss which was associated with an impairment of her short-term memory. A computed tomographic (CT) head scan was performed, the appearance of which was consistent with a cerebral glioblastoma arising in the left parietal lobe.

On examination the following day, she was normally oriented and had minimal weakness of the right arm and leg. There was no perception of light bilaterally, the pupils were equal and unresponsive, and the ocular movements were full. The remainder of the ocular examination was normal; in particular, the optic discs were neither swollen nor atrophic.

As the visual loss was bilateral, symmetrical and pupil-involving, the lesion was localised to the optic chiasm. The rapidity of the visual deterioration suggested the underlying cause was acute vascular insufficiency. The patient had no symptoms suggestive of giant cell arteritis and the ESR was $41 \mathrm{~mm} / \mathrm{h}$. The magnetic resonance (MR) image is shown in Fig. 1.

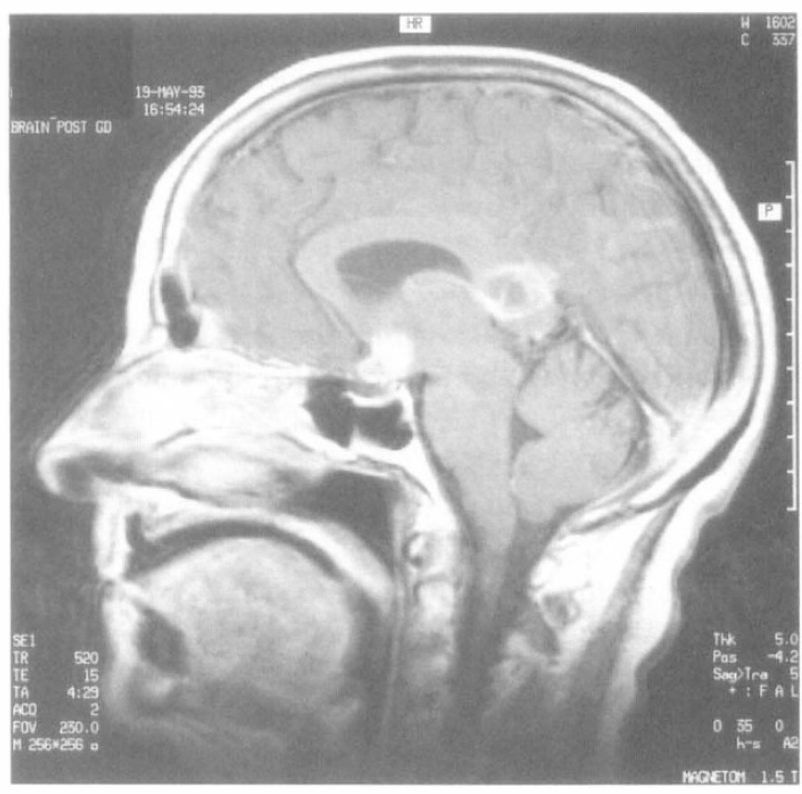

Fig. 1. Sagittal, midline, T1-weighted, post-gadolinium MR scan demonstrating an enhancing cystic mass in the splenium of the corpus callosum. A separate enhancing focus arises from the floor of the third ventricle and assumes the configuration of the chiasmatic and infundibular recesses. Within this focus lies an area of low-density signal that is iso-intense with that of brain and is consistent with infarction of the optic chiasm. The pre-contrast images (not shown) demonstrate the presence of perichiasmal haemorrhage. 